\title{
Synthesis of two 3,5-disubstituted sulfonamide catechol ligands and evaluation of their iron(III) complexes for use as MRI contrast agents
}

Daniel D. Schwert, ${ }^{\dagger}, *$ Nicholas Richardson, ${ }^{\star}$ Gongjun Ji, ${ }^{\dagger}$ Bernd Radüchel,${ }^{\infty}$ Wolfgang Ebert $^{\infty}$ Peter E. Heffner ${ }^{\dagger}$ Rick Keck, ${ }^{\#}$ and Julian A. Davies ${ }^{\dagger}$

$\dagger$ Department of Chemistry, University of Toledo, $2801 \mathrm{~W}$ Bancroft St, Toledo, OH, 43606

† Department of Chemistry and Physics, Wagner College, Staten Island, NY 10301

$\infty$ Research Laboratories of Schering AG, 13342 Berlin, Germany

\# Department of Urology, Medical College of Ohio, Toledo, OH, 43614

Elemental Analysis Data For All Target Compounds

\begin{tabular}{|c|c|c|c|c|}
\hline Compound Name & $\begin{array}{c}\text { Compound } \\
\text { Number }\end{array}$ & $\begin{array}{l}\text { Found \% C } \\
\text { (calculated) }\end{array}$ & $\begin{array}{l}\text { Found \% H } \\
\text { (calculated) }\end{array}$ & $\begin{array}{l}\text { Found \% N } \\
\text { (calculated) }\end{array}$ \\
\hline $\begin{array}{c}\text { 3,5-Bis(diethylsulfonamide) } \\
\text { catechol }\end{array}$ & $\mathrm{H}_{2} 2$ & $\begin{array}{c}44.57 \\
(44.19)\end{array}$ & $\begin{array}{c}6.47 \\
(6.36)\end{array}$ & $\begin{array}{c}7.07 \\
(7.36)\end{array}$ \\
\hline $\begin{array}{c}3,5- \\
\text { Bis(dimethylsulfonamide) } \\
\text { catechol }\end{array}$ & $\mathrm{H}_{2} 3$ & $\begin{array}{c}37.04 \\
(37.03)\end{array}$ & $\begin{array}{c}4.99 \\
(4.97)\end{array}$ & $\begin{array}{c}8.49 \\
(8.64)\end{array}$ \\
\hline $\begin{array}{c}\text { Trisodium tris }[3,5- \\
\text { bis(diethylsulfonamide) } \\
\text { catecholato iron(III) } \\
\text { heptahydrate }\end{array}$ & $\begin{array}{c}\mathrm{Na}_{3} \mathrm{Fe}(2)_{3} \\
\quad 7 \mathrm{H}_{2} \mathrm{O}\end{array}$ & $\begin{array}{c}36.49 \\
(36.39)\end{array}$ & $\begin{array}{c}5.64 \\
(5.82)\end{array}$ & $\begin{array}{c}5.93 \\
(6.06)\end{array}$ \\
\hline $\begin{array}{c}\text { Trisodium tris }[3,5- \\
\text { bis(dimethylsulfonamide) } \\
\text { catecholato iron(III) } \\
\text { heptahydrate }\end{array}$ & $\begin{array}{c}\mathrm{Na}_{3} \mathrm{Fe}(3)_{3} \\
7 \mathrm{H}_{2} \mathrm{O}\end{array}$ & $\begin{array}{c}29.28 \\
(29.58)\end{array}$ & $\begin{array}{c}4.46 \\
(4.63)\end{array}$ & $\begin{array}{c}6.88 \\
(6.90)\end{array}$ \\
\hline
\end{tabular}

